\title{
Cardiac Rehabilitation: The Best Rehabilitation Has to Offer
}

\author{
Ioan-Sorin Stratulat ${ }^{1,2}$, Ioana-Alexandra Benea ${ }^{3}$ \\ ${ }^{1}$ Associate Prof. of Rehabilitation Medicine of Dental Medicine, University of Medicine and Pharmacy „Gr.T.Popa” and \\ ${ }^{2}$ Head of the Department of Clinical Rehabilitation Medicine, C.F. Hospital Iasi, Romania (E-mail: dr_sorin_stratulat@yahoo.com) \\ ${ }^{3}$ Young Resident of Rehabilitation Medicine, Clinical Rehabilitation Hospital Iasi, Romania
}

\begin{abstract}
Introduction

Over the last decade, cardiac rehabilitation has gained a lot of interest and widespread acceptance and plays an important role in the outcome of the ischemic heart disease. Cardiac conditions frequently lead to limited exercise capacity, physical disability and poor quality of life if rehabilitation programmes are not instituted promptly. A well designed cardiac rehabilitation programme should include some important aspects, such as: a precise clinical evaluation, adapted pharmacotherapy, up-to-date physical training and last, but not least, good psychological support.

Objectives

In order to obtain good results and to develop a high standard rehabilitation programme, there is a need to create a professional, multidisciplinary team, that will include: physicians, nurses, dietician, social workers, psychotherapists. Moreover, the training programme should start as soon as possible after an acute phase of myocardial infarction, with individualized schedule designed to optimize physical, social and emotional status.

Methods

Prior to the programme commencing, patients admitted will benefit from a proper, complete medical evaluation and a graded exercise tolerance test, to establish the necessary steps that need to be taken in order to obtain an optimal recovery. Close supervision is needed when the exercises are performed and also, dynamic exercises are preferred over isometrics. There are multiple phases (1A,1B,2,3), each one with its own particularities and objectives. The hardest of them is Phase 3Maintenance, due to the fact that benefits of phase 2 can be immediately lost ( a few weeks) if regular exercise is not maintained properly, as well as the other recommendations. Patients that can benefit from this type of programme include: the ones that underwent bypass surgery, after heart failure, valvular disease, stroke, pre and post-heart transplant, LVAD (Left Ventricular Assist Device).

Results

Each phase of the programme has good results, with a substantial improvement in the clinical status of the patient, but there is still room for changes. In phase 3 , if we do not offer proper medical training to the patient- in order for him to fully comprehend the benefits of rigorously continuing each step of the programme-, the clinical improvements can easily be lost. Some of the major results are: a good blood pressure control, lipid control, physical resistance and decreased symptoms associated with depression.

Conclusions

To conclude with, a very important aspect to remember is that rehabilitating programmes represent the future in maintaining a proper social and healthy life, for patients that underwent some major cardiac changes throughout their lives. Ultimate comprehensive cardiac rehabilitation needs to be immediately initiated, properly adapted for each patient's clinical status and staged and also, continued for required time. We must improve existing programmes and we have to develop new ones, but only if we collaborate and create multidisciplinary teams, in order to obtain the maximum benefit for the patient.
\end{abstract}

Keywords: Cardiac Rehabilitation, Multidisciplinary Teams, Maintenance 